Rev. Bras. Saúde Prod. Anim., Salvador, v.16, n.3, p.667-677 jul./set.., $2015 \quad \underline{\text { http://www.rbspa.ufba.br }}$

\title{
Jejum alimentar e qualidade da carne de frango de corte tipo caipira
}

\author{
Pre slaughter fasting and free-range broilers meat quality
}

\author{
OLIVEIRA, Felipe Rosa ${ }^{1 *}$; BOARI, Cleube Andrade ${ }^{2}$; PIRES, Aldrin Vieira ${ }^{2}$; \\ MOGNATO, João Carlos ${ }^{3}$; CARVALHO, Rúbio Madureira de Souza ${ }^{1}$; SANTOS \\ JÚNIOR, Marco Aurélio ${ }^{3}$; MATTIOLI, Cristiano Campos ${ }^{4}$
}

\footnotetext{
${ }^{1}$ Universidade Federal dos Vales do Jequitinhonha e Mucuri, Programa de Pós-Graduação em Zootecnia, Diamantina, Minas Gerais, Brasil.

${ }^{2}$ Universidade Federal dos Vales do Jequitinhonha e Mucuri, Departamento de Zootecnia, Diamantina, Minas Gerais, Brasil.

${ }^{3}$ Universidade Federal dos Vales do Jequitinhonha e Mucuri, Diamantina, Minas Gerais, Brasil.

${ }^{4}$ Universidade Federal de Minas Gerais, Programa de Pós-Graduação em Zootecnia, Belo Horizonte, Minas Gerais, Brasil.

*Endereço para correspondência: feliperosa610@gmail.com
}

\section{RESUMO}

Objetivou-se avaliar as características de qualidade da carne de frango de corte tipo caipira em diferentes tempos de jejum alimentar. Aves, machos, da linhagem "pesadão vermelho", criadas até 85 dias de idade em sistema semiextensivo, foram submetidas aos tempos de zero, três, seis, nove e 12 horas de jejum alimentar. Obteve-se o peso vivo, peso da carcaça, o rendimento de carcaça fria, o peso do trato gastrintestinal das aves. Foram avaliados o $\mathrm{pH}_{24 \mathrm{~h}}$, a luminosidade $\left(L^{*}\right)$, o teor de vermelho $\left(a^{*}\right)$, o teor de amarelo $\left(b^{*}\right)$, a capacidade de retenção de água, a perda de peso por cocção e a força de cisalhamento na carne do peito, da coxa e da sobrecoxa. Menor valor de rendimento de carcaça foi observado no tempo zero hora $(74,54 \%)$, e o maior $(82,43 \%)$ às nove horas de jejum alimentar. Maior peso para o trato gastrintestinal $(540,8 \mathrm{~g})$ foi observado no tempo zero e o menor $(229,33 \mathrm{~g})$ às 12 horas de jejum alimentar. Não foi observado efeito dos diferentes tempos de jejum alimentar nas características de qualidade da carne das aves. Houve diferença no $\mathrm{pH}_{24 \mathrm{~h}}$, na luminosidade $\left(L^{*}\right)$, no teor de vermelho $\left(a^{*}\right)$, no teor de amarelo $\left(b^{*}\right)$ e na capacidade de retenção de água quando se comparou os cortes da carne do peito, da coxa e da sobrecoxa das aves de corte tipo caipira. Recomendam-se os períodos de jejum de nove ou 12 horas, nos quais houve maior rendimento de carcaça fria.

Palavras-chave: coxa, força de cisalhamento, peito, $\mathrm{pH}_{24 \mathrm{~h}}$, sobrecoxa

\section{SUMMARY}

It aimed to evaluate the quality characteristics of the rustic type broiler meat at different times of fasting. Birds, males, Pesadão Vermelho lineage, created up to 85 days old in semiextensivo system, were subject to the zero times, three, six, nine and 12 hours of fasting. This gave the live weight, carcass weight, cold carcass yield, the weight of the gastrointestinal tract of birds. The $\mathrm{pH}_{24 \mathrm{~h}}$ were evaluated, lightness $\left(L^{*}\right)$, red content $\left(a^{*}\right)$, a yellow content $\left(b^{*}\right)$, the water holding capacity, cooking loss of weight and shear force in meat breast, thigh and drumstick. Carcass yield lower was observed in the zero hour $(74.54 \%)$ and the largest $(82.43 \%)$ to nine hours of fasting. Greater weight to the gastrointestinal tract $(540,8 \mathrm{~g})$ was observed at time zero and the lowest $(229,33 \mathrm{~g})$ at 12 hours of fasting. There was no effect of different times of fasting in the quality characteristics of the meat of birds. There was a difference in $\mathrm{pH}_{24 \mathrm{~h}}$, the lightness $\left(L^{*}\right)$, red content $\left(a^{*}\right)$, yellow content $\left(b^{*}\right)$ and water holding capacity when compared the breast meat cuts, thigh and drumstick of the rustic type broiler. Periods of fasting are recommended nine or 12 hours, in which there was higher cold carcass yield.

Keywords: breast, drumstick, $\mathrm{pH}_{24 \mathrm{~h}}$, shear force, thigh 
Rev. Bras. Saúde Prod. Anim., Salvador, v.16, n.3, p.667-677 jul./set.., $2015 \quad \underline{\text { http://www.rbspa.ufba.br }}$

\section{INTRODUÇÃO}

O jejum alimentar, etapa primordial para o abate, inicia-se na granja com a suspensão da alimentação das aves, preservando-se o fornecimento de água potável até o instante da apanha para o encaminhamento ao abatedouro (CASTRO et al., 2008; PEREIRA et.al., 2013). Sua finalidade consiste em diminuir o volume de conteúdo do trato gastrintestinal para reduzir as possibilidades de rompimento de alças intestinais e a consequente condenação da carcaça por contaminação cruzada (KOMIYAMA et. al., 2008; RAMÃO et.al., 2011; PEREIRA, 2013; BARACHO et. al., 2012).

O tempo de jejum alimentar pode influenciar na disponibilidade de glicogênio post mortem, que será metabolizado no músculo. A extensão de acidificação muscular influencia no $\mathrm{pH}$ final, o qual, por sua vez, também influenciará na luminosidade e cor da carne, e na sua capacidade de retenção de água, na perda de peso por cozimento e na força de cisalhamento (STERTEN et al., 2009).

A inadequada acidificação, ocasionada pelo estresse, é a causa para a ocorrência das carnes anômalas com corte seco, de coloração escura, com baixa luminosidade e firme (DFD: $\mathrm{pH}>$ $5,8)$, de aparência pálida, textura flácida e exsudativa $(\mathrm{pH} \sim \mathrm{pI}$ proteínas miofibrilares 5,2) (WARRIS, 2010). Estas carnes anômalas podem acarretar prejuízos diversos devido a sua inadequada aptidão tecnológica ao processamento agroindustrial e aos problemas decorrentes de sua aceitação pelo consumidor.

Por esta razão, a influência do jejum alimentar sobre a qualidade da carne é motivo de preocupação para o setor produtivo e deve ser programado de acordo com as necessidades dos frigoríficos a fim de se garantir a qualidade dos derivados cárneos (SCHETTINO et al., 2006).

Não há uma legislação específica para abate de frangos tipo caipira e, por isto, é utilizada a recomendação para frangos de corte criados em sistema convencional, o qual varia de seis a 12 horas de jejum, dependendo da distância entre a granja e abatedouro e das condições de transporte (CASTRO et al., 2008).

Dentro deste contexto, o objetivo deste estudo foi avaliar a qualidade da carne de frango de corte tipo caipira submetido a diferentes períodos de jejum alimentar.

\section{MATERIAL E MÉTODOS}

Este experimento foi conduzido nos setores de Avicultura e Ciência e tecnologia dos produtos de origem animal, do departamento de Zootecnia, da Universidade Federal dos Vales do Jequitinhonha e Mucuri, Diamantina, Minas Gerais, durante o mês de maio do ano de 2011.

O delineamento experimental utilizado foi inteiramente casualizado, com cinco tratamentos que corresponderam aos tempos de jejum alimentar (zero, três, seis, nove e 12 horas) e cinco repetições. Após completar 85 dias de idade, as aves $(\mathrm{n}=25)$ foram, aleatoriamente, alojadas em cinco boxes (2,00x 1,50m), específicos para cada um dos cinco tempos de jejum alimentar. $\mathrm{O}$ acesso dos animais à água potável foi assegurado até o momento da apanha para abate.

Machos da linhagem "pesadão vermelho" foram confinados até os 25 dias de idade e, posteriormente, tiveram acesso livre a piquete gramado com 
Rev. Bras. Saúde Prod. Anim., Salvador, v.16, n.3, p.667-677 jul./set.., $2015 \quad \underline{\text { http://www.rbspa.ufba.br }}$ ISSN 15199940

Tifton $\left(45 \mathrm{~m}^{2}\right)$. As dietas experimentais foram formuladas de modo a atender as exigências nutricionais das aves, para cada fase de crescimento (ROSTAGNO el al., 2005), conforme apresentado na Tabela 1.

As aves foram apanhadas pelo dorso, acondicionadas em gaiolas e transportadas à sala de abate experimental, a qual é iluminada por luz artificial azul, onde foram pesadas, insensibilizadas por deslocamento cervical, procedendo-se, imediatamente, à sangria, seguida por intervalo de 3 minutos. Conduziu-se a depenagem, evisceração, toalete da carcaça, préresfriamento em água clorada a $15^{\circ} \mathrm{C}$ por 20 minutos e resfriamento $\mathrm{em}$ câmara fria $\left(0^{\circ} \mathrm{C} \pm 1\right)$.

Tabela 1. Composições das rações nas três fases de criação das aves

\begin{tabular}{|c|c|c|c|}
\hline Ingredientes $(\%)$ & 1 a 28 dias & 28 a 56 dias & 56 a 84 dias \\
\hline Milho Moído & 56,792 & 62,518 & 71,793 \\
\hline Farelo de Soja $45 \%$ & 36,302 & 30,822 & 24,089 \\
\hline Inerte (areia fina) & 2,558 & 3,000 & 0,914 \\
\hline Fosfato Bicálcico & 2,044 & 1,327 & 1,212 \\
\hline Calcário & 1,429 & 1,196 & 1,094 \\
\hline Sal comum & 0,457 & 0,331 & 0,254 \\
\hline DL- Metionina & 0,217 & 0,174 & 0,260 \\
\hline Suplemento Vitamínico ${ }^{1}$ & 0,100 & 0,100 & 0,100 \\
\hline Suplemento Mineral ${ }^{2}$ & 0,100 & 0,100 & 0,100 \\
\hline Óleo de Soja & 0,000 & 0,311 & 0,184 \\
\hline L- Lisina HCL & 0,000 & 0,120 & 0,000 \\
\hline Total & 100,00 & 100,00 & 100,00 \\
\hline \multicolumn{4}{|l|}{ Composição química calculada } \\
\hline Proteína Bruta (\%) & 21,27 & 19,35 & 17,00 \\
\hline Energia Metabolizável (kcal/kg) & 2750 & 2850 & 3000 \\
\hline Lisina digestível (\%) & 1,04 & 1,00 & 0,76 \\
\hline Metionina digestível (\%) & 0,51 & 0,44 & 0,50 \\
\hline Metionina + Cistina digestível $(\%)$ & 0,80 & 0,71 & 0,75 \\
\hline Cálcio $(\%)$ & 1,16 & 0,88 & 0,80 \\
\hline Fósforo disponível (\%) & 0,48 & 0,35 & 0,32 \\
\hline
\end{tabular}

${ }^{1}$ Suplemento vitamínico por kg de produto: vit. A 3.750.000 UI; vit. D3, 750.000 UI; vit. E 7500mg; vit K3, 1.000mg; vit. B1, 750mg; vit. B2, 1.500mg; vit. B6, $1500 \mathrm{mg}$; vit. B12, $7.500 \mathrm{mcg}$; vit. C $12.500 \mathrm{mg}$, biotina $30 \mathrm{mg}$, niacina $10.000 \mathrm{mg}$, ácido fólico 375 ; acid pantotênico $3.750 \mathrm{mg}$; colina $10.000 \mathrm{mg}$, metionina $400.000 \mathrm{mg}$.

${ }^{2}$ Suplemento mineral por kg de produto: selênio $45 \mathrm{mg}$; iodo $175 \mathrm{mg}$; ferro $12.525 \mathrm{mg}$; cobre $2.500 \mathrm{mg}$; manganês, $19.500 \mathrm{mg}$; zinco $13.750 \mathrm{mg}$; prom. Prod $15.000 \mathrm{mg}$, coccidiostático $10.000 \mathrm{mg}$, antioxidante (B.H.T) $500 \mathrm{mg}$.

Para fins de cálculos de rendimento da carcaça, obteve-se o peso da carcaça fria (com cabeça e pés) e o peso vivo; em seguida, pesou-se o trato gastrointestinal. Os parâmetros $\mathrm{pH}_{24 \mathrm{~h}}$, componentes da cor $\left(L^{*} a * b^{*}\right)$, capacidade de retenção de água, força de cisalhamento e perda de peso por cocção foram mensurados nos cortes representados pelo peito, coxa e sobrecoxa, 24 horas após resfriamento.

Obteve-se o $\mathrm{pH}_{24 \mathrm{~h}}$ (média de 3 leituras) por uso de pHmetro acoplado com eletrodo de penetração (Hanna Instruments). Luminosidade $\left(L^{*}\right)$, teor de vermelho $\left(a^{*}\right)$ e amarelo $\left(b^{*}\right)$ foram diretamente mensurados na carne, utilizando-se colorímetro (Minolta CR- 
Rev. Bras. Saúde Prod. Anim., Salvador, v.16, n.3, p.667-677 jul./set.., $2015 \quad \underline{\text { http://www.rbspa.ufba.br }}$ ISSN 15199940

400), no sistema CIELab, sendo os valores de cada repetição, calculados a partir da média de 3 leituras (VAN LAACK et al., 2000). A capacidade de retenção de água foi determinada segundo a metodologia de Yancey \& Apple (2011). A perda de peso por cocção foi determinada conforme $\mathrm{o}$ procedimento descrito por Barge et al. (1991). A força de cisalhamento foi determinada com texturômetro (modelo TA.XT2 plus ${ }^{\circledR}$, Stable micro system, Godalming, Surrey, Reino Unido), acoplado com sonda de cisalhamento retangular padrão Warner-Bratzler, com espessura de $1,016 \mathrm{~mm}$ e com lâmina de $3,05 \mathrm{~mm}$.

Os dados (picos positivos máximos) foram obtidos com o programa Exponent Lite versão 5.1 (Stable micro systems). O equipamento foi calibrado com peso padrão de $5 \mathrm{~kg}$, sendo programado com velocidade de descida e de corte do dispositivo de 200mm.minuto ${ }^{-1}$, velocidade de pré-teste, de teste e de pósteste de $2 \mathrm{~mm}$. segundo $^{-1}$ e distância de penetração de $15 \mathrm{~mm}$. Para esta análise, foram utilizadas amostras remanescentes da análise da perda de peso por cozimento, as quais foram resfriadas $\left(4^{\circ} \mathrm{C}\right)$ por 24 horas e, assim, mantidas até o momento das análises. Foram retiradas subamostras em forma de paralelepípedos 1x1x2cm (altura, largura e comprimento, respectivamente), sendo dispostas no equipamento com as fibras orientadas no sentido perpendicular à probe. Os resultados foram expressos $\mathrm{kgf.cm}{ }^{-2}$ (AMSA, 1995).

Os dados das análises dos diferentes tempos de jejum alimentar foram submetidos à análise de variância e análise de regressão. As médias dos valores dos cortes do peito, da coxa e da sobrecoxa foram comparadas por meio de contraste ortogonal utilizando-se o teste de Tukey, em nível de 5\% significância. As análises estatísticas forma realizadas utilizando-se o programa Statistical analysis system versão 6.0 (SAS, 1996).

O projeto experimental foi aprovado pela Comissão de ética e no uso de animais da UFVJM (Protocolo de registro 015/10).

\section{RESULTADOS E DISCUSSÃO}

Houve efeito significativo $(\mathrm{P}<0,001)$ para rendimento de carcaça e peso do trato gastrointestinal (Tabela 2), em resposta aos diferentes períodos de jejum alimentar.

Tabela 2. Peso vivo ao abate, peso da carcaça, rendimento de carcaça e peso do tratogastrointestinal de aves tipo caipira da linhagem pesadão vermelho abatidas após diferentes períodos de jejum alimentar

\begin{tabular}{lcccc}
\hline \multicolumn{5}{c}{ Parâmetros } \\
\hline Tempo de jejum & PV $(\mathrm{g})^{1}$ & PC $(\mathrm{g})^{2}$ & RC $(\%)^{3} * * *$ & TGI $(\mathrm{g})^{4} * *$ \\
\hline 0 & $4363,60 \pm 615,37$ & $3261,60 \pm 554,37$ & $73,58 \pm 1,01$ & $540,80 \pm 66,29$ \\
3 & $4476,40 \pm 556,76$ & $3438,00 \pm 405,15$ & $78,54 \pm 1,34$ & $371,75 \pm 147,33$ \\
6 & $4074,00 \pm 213,41$ & $3134,00 \pm 228,72$ & $76,87 \pm 2,70$ & $279,50 \pm 30,17$ \\
9 & $4431,60 \pm 240,58$ & $3652,00 \pm 172,42$ & $82,43 \pm 1,48$ & $272,20 \pm 19,79$ \\
12 & $4394,80 \pm 533,59$ & $3568,00 \pm 477,16$ & $81,37 \pm 1,34$ & $229,33 \pm 12,05$ \\
CV (\%) & 10,67 & 11,58 & 2,02 & 22,59 \\
\hline
\end{tabular}

${ }^{1}$ peso vivo ao abate; ${ }^{2}$ peso da carcaça; ${ }^{3}$ rendimento de carcaça; ${ }^{4}$ peso do trato gastrintestinal.

$* * * \mathrm{P}<0,001\left(\mathrm{y}=-0,0736 \mathrm{x}^{2}+1,50646 \mathrm{x}+73,83711 \mathrm{r}^{2}=0,8020\right)$.

$* * \mathrm{P}<0,01\left(\mathrm{y}=-53,31293 \mathrm{x}+527,2891 \mathrm{r}^{2}=0,6674\right)$. 
Rev. Bras. Saúde Prod. Anim., Salvador, v.16, n.3, p.667-677 jul./set.., $2015 \quad \underline{\text { http://www.rbspa.ufba.br }}$

O menor rendimento de carcaça foi observado no tempo de zero hora de jejum, o que foi ocasionado pelo maior peso do trato gastrointestinal devido à maior quantidade de digesta (ASSAYAG et al., 2005; GARCIA et al., 2008). O maior rendimento de carcaça foi observado às nove horas de jejum, tempo no qual também se observou que $\mathrm{o}$ peso do trato gastrointestinal foi significativamente menor $(\mathrm{P}<0,001)$. No entanto, com o avançar do período por mais de nove horas observou-se redução no rendimento de carcaça.

Ramão et al. (2011) observaram que os rendimentos de carcaça quente e de carcaça fria foram influenciados pelo tempo de duração do jejum alimentar, sendo estes, linearmente maiores quanto maior foi o tempo de jejum alimentar. Assim como observado nesta pesquisa, os referidos autores também observaram menor rendimento de carcaça fria nas aves que não foram submetidas ao jejum alimentar.

Mendes (2001) observou efeito significativo do período de jejum sobre o rendimento de carcaça de aves da linhagem Ross, sendo obtido maior rendimento para os períodos de quatro horas $(69,21 \%)$ e oito horas $(69,23 \%)$ de jejum alimentar. Castro et al. (2008) observaram maior rendimento de carcaça de frangos de corte entre os períodos de três horas $(67,44 \%)$ e seis horas $(67,42 \%)$, seguido de queda gradativa a partir de nove horas $(66,16 \%)$ de jejum. O peso do trato gastrointestinal foi significativamente $(\mathrm{P}<0,01)$ maior com zero hora de jejum alimentar, seguido da diminuição de seu peso a partir da zero hora.

Os períodos de jejum alimentar não influenciaram $(\mathrm{P} \geq 0,01) \quad \mathrm{o} \quad \mathrm{pH}_{24 \mathrm{~h}}, \quad \mathrm{a}$ luminosidade $\left(L^{*}\right)$, o teor de vermelho $\left(a^{*}\right)$, o teor de amarelo $\left(b^{*}\right)$, a capacidade de retenção de água, a força de cisalhamento e a perda de peso por cocção do peito, coxa e sobrecoxa (Tabela 3). As variações não significativas na cor $\left(L^{*} a^{*} b^{*}\right)$, na força de cisalhamento, na capacidade de retenção de água e na perda de peso por cocção destes cortes, provavelmente, decorrem da não significância nas variações do $\mathrm{pH}_{24 \mathrm{~h}}$, tendo-se em vista a dependência entre estes parâmetros (STERTEN et al., 2009).

Os valores de $\mathrm{pH}_{24 \mathrm{~h}}$ do peito variaram entre 5,61 e 5,7, sendo inferiores a 5,8, valor a partir do qual há possibilidade da ocorrência da carne DFD, superiores, porém, ao valor do ponto isoelétrico ( $\mathrm{pI}$ $\sim 5,2$ ) das proteínas miofibrilares, no qual há possibilidade de ocorrência de carne PSE (WARRIS, 2010). Assim como observado nesta pesquisa, Denadai et al. (2002), Komiyama et al. (2008) e Pereira (2013) também não observaram efeito significativo do tempo de jejum pré-abate sobre o $\mathrm{pH}$ final da carne de peito de aves.

Diferente dos dados observados neste estudo, Komiyama et al. (2008) relataram efeitos significativos de diferentes períodos de jejum alimentar na luminosidade $\left(L^{*}\right)$, no teor de vermelho $\left(a^{*}\right)$, na força de cisalhamento e na capacidade de retenção de água da carne do peito de frangos machos da linhagem Ross. Entretanto, há de se ressaltar que os valores de $\mathrm{pH}$ final observados por estes autores foram superiores aos observados no presente estudo. Almejando-se a produção de carne com qualidade, Komiyama et al. (2008) recomendaram o tempo de jejum alimentar de pelo menos oito horas, sendo este, sugerido como necessário para que as aves se recuperem do estresse ocasionado pelo manejo préabate. 
Tabela 3. Características de qualidade da carne de frango tipo caipira abatidas após diferentes períodos de jejum alimentar

\begin{tabular}{|c|c|c|c|c|c|c|c|c|}
\hline Item & Tempo & $\mathrm{pH}_{24 \mathrm{~h}}$ & $L^{*}$ & $a^{*}$ & $b^{*}$ & CRA (\%) & $\mathrm{FC}\left(\mathrm{kgf.cm}{ }^{-2}\right)$ & PPC (\%) \\
\hline \multirow{5}{*}{ Peito } & 0 & $5,61 \pm 0,11$ & $59,20 \pm 4,63$ & $0,77 \pm 1,17$ & $4,19 \pm 2,98$ & $56,94 \pm 3,99$ & $1,77 \pm 0,76$ & $28,42 \pm 3,36$ \\
\hline & 3 & $5,7 \pm 0,16$ & $58,88 \pm 2,97$ & $0,11 \pm 0,42$ & $6,41 \pm 1,76$ & $58,76 \pm 8,02$ & $2,00 \pm 0,0,85$ & $24,78 \pm 4,12$ \\
\hline & 6 & $5,65 \pm 0,07$ & $59,27 \pm 2,91$ & $0,21 \pm 0,49$ & $3,36 \pm 1,48$ & $54,55 \pm 2,15$ & $1,97 \pm 0,82$ & $25,63 \pm 4,80$ \\
\hline & 9 & $5,67 \pm 0,08$ & $57,68 \pm 3,35$ & $0,08 \pm 0,71$ & $3,95 \pm 1,87$ & $57,62 \pm 2,93$ & $2,15 \pm 0,65$ & $25,85 \pm 2,70$ \\
\hline & 12 & $5,71 \pm 0,12$ & $58,85 \pm 2,57$ & $0,99 \pm 1,41$ & $3,39 \pm 3,34$ & $55,78 \pm 2,02$ & $1,71 \pm 0,68$ & $23,45 \pm 4,31$ \\
\hline $\mathrm{CV}(\%)$ & & 1,96 & 5,53 & 33,33 & 58,60 & 7,96 & 31,17 & 15,22 \\
\hline \multirow{5}{*}{ Coxa } & 0 & $5,81 \pm 0,13$ & $59,53 \pm 4,71$ & $6,41 \pm 2,23$ & $5,99 \pm 3,20$ & $59,43 \pm 3,34$ & $2,24 \pm 0,95$ & $34,68 \pm 5,10$ \\
\hline & 3 & $5,87 \pm 0,12$ & $56,13 \pm 3,53$ & $8,41 \pm 2,10$ & $8,68 \pm 1,84$ & $54,22 \pm 3,84$ & $1,62 \pm 0,57$ & $30,98 \pm 2,20$ \\
\hline & 6 & $5,80 \pm 0,05$ & $59,67 \pm 5,24$ & $7,26 \pm 4,01$ & $4,97 \pm 4,46$ & $54,71 \pm 5,42$ & $2,26 \pm 0,84$ & $33,79 \pm 4,87$ \\
\hline & 9 & $5,91 \pm 0,11$ & $56,17 \pm 2,35$ & $9,36 \pm 2,90$ & $9,07 \pm 2,41$ & $55,09 \pm 7,64$ & $2,01 \pm 0,70$ & $33,71 \pm 2,60$ \\
\hline & 12 & $5,88 \pm 0,06$ & $59,53 \pm 5,70$ & $8,22 \pm 5,20$ & $8,04 \pm 4,97$ & $59,87 \pm 2,40$ & $1,62 \pm 0,59$ & $30,08 \pm 7,42$ \\
\hline CV $(\%)$ & & 1,67 & 7,82 & 42,85 & 51,02 & 7,96 & 33,48 & 14,91 \\
\hline \multirow{5}{*}{ Sobrecoxa } & 0 & $5,81 \pm 0,10$ & $54,39 \pm 3,36$ & $6,83 \pm 2,10$ & $4,72 \pm 3,81$ & $56,05 \pm 1,78$ & $2,32 \pm 0,70$ & $36,32 \pm 3,09$ \\
\hline & 3 & $5,75 \pm 0,12$ & $53,06 \pm 1,30$ & $7,89 \pm 2,55$ & $9,22 \pm 0,56$ & $54,32 \pm 3,67$ & $1,82 \pm 0,57$ & $34,81 \pm 5,57$ \\
\hline & 6 & $5,81 \pm 0,07$ & $56,65 \pm 5,52$ & $8,75 \pm 3,72$ & $7,04 \pm 3,42$ & $53,92 \pm 3,10$ & $2,32 \pm 0,73$ & $36,76 \pm 3,12$ \\
\hline & 9 & $5,89 \pm 0,05$ & $52,24 \pm 2,66$ & $10,23 \pm 1,80$ & $8,47 \pm 1,94$ & $54,44 \pm 2,40$ & $1,44 \pm 0,52$ & $32,55 \pm 4,45$ \\
\hline & 12 & $5,90 \pm 0,12$ & $55,85 \pm 5,24$ & $7,74 \pm 2,98$ & $6,41 \pm 2,52$ & $53,85 \pm 6,43$ & $1,71 \pm 0,48$ & $32,69 \pm 6,87$ \\
\hline CV (\%) & & 1,67 & 7,60 & 32,18 & 39,17 & 6,82 & 31,51 & 13,74 \\
\hline
\end{tabular}

$L^{*}=$ Luminosidade; $a^{*}=$ teor de vermelho; $b^{*}=$ teor de amarelo; $\mathrm{CRA}=$ capacidade de retenção de água; $\mathrm{FC}=$ força de cisalhamento; PPC $=$ perda de peso por cocção. 
Rev. Bras. Saúde Prod. Anim., Salvador, v.16, n.3, p.667-677 jul./set.., $2015 \quad \underline{\text { http://www.rbspa.ufba.br }}$ ISSN 15199940

Conforme observado nesta pesquisa, Pereira et al. (2013) também verificaram que os tempos de jejum alimentar (seis, nove, 12 e 15) não afetaram $(\mathrm{P} \geq 0,05) \mathrm{o}$ $\mathrm{pH}$, o teor de vermelho $\left(a^{*}\right)$ e o teor de amarelo $\left(b^{*}\right)$ da carne do peito de frangos de corte (machos) da linhagem Cobb. Entretanto, os referidos autores relataram efeito significativo do tempo de jejum na luminosidade $\left(L^{*}\right)$, mas justificaram que, mesmo havendo variação, os valores observados são considerados como normais para a carne do peito de frangos de corte (PSE $L^{*}>53$; DFD $L^{*}<46$; Normal $\left.46<L^{*}<53\right)$.

Confrontando-se o intervalo de luminosidade $\left(L^{*}\right)$, mencionado por Pereira et al. (2013) como sendo normal, para linhas de frangos de corte de crescimento rápido, com os dados desta pesquisa, observou-se que os frangos de corte de crescimento lento, em condições de $\mathrm{pH}$ normal, apresentaram luminosidade superior ao limite máximo $\left(L^{*}>53\right)$. Este evento pode ser justificado pelas diferenças intrínsecas da estrutura e composição muscular entre frangos de corte de crescimento rápido e lento.
A capacidade de retenção de água, a perda de peso por cozimento e a força de cisalhamento são parâmetros de qualidade da carne, influenciáveis pelo pH (STERTEN et al., 2009; WARRIS, 2010). Portanto, as variações não significativas destes parâmetros, nos diferentes tempos de jejum alimentar, podem ser explicados pelas variações não significativas do $\mathrm{pH}_{24 \mathrm{~h}}$. Para frangos Ross, Komiyama et al. (2008) verificaram, em crescentes períodos de jejum alimentar, aumentos na capacidade de retenção de água e na força de cisalhamento da carne, os quais, ocasionados pelo aumento do $\mathrm{pH}$. Comparando-se as características de qualidade entre os cortes pesquisados, verificou-se que a carne do peito apresentou $\mathrm{pH}_{24 \mathrm{~h}}$ significativamente inferior $(\mathrm{P}<0,05)$ que a carne da coxa $\mathrm{e}$ da sobrecoxa (Tabela 4). Houve, também, diferença significativa $(\mathrm{P}<0,05)$ na cor $\left(L^{*} a^{*} b^{*}\right)$ e na capacidade de retenção de água da carne. Não houve diferença significativa $(\mathrm{P} \geq 0,05)$ na força de cisalhamento e na perda de peso por cocção.

Tabela 4. Características de qualidade da carne de frango tipo caipira, abatida após diferentes períodos de jejum alimentar

\begin{tabular}{lcccc}
\hline \multicolumn{5}{c}{ Corte cárneo } \\
\hline Característica & Peito & Coxa & Sobrecoxa & CV $(\%)$ \\
\hline $\mathrm{pH}_{24 \mathrm{~h}}$ & $5,66 \pm 0,04^{\mathrm{b}}$ & $5,85 \pm 0,04^{\mathrm{a}}$ & $5,83 \pm 0,06^{\mathrm{a}}$ & 0,89 \\
$L^{*}$ & $58,77 \pm 0,64^{\mathrm{a}}$ & $56,20 \pm 1,87^{\mathrm{b}}$ & $54,43 \pm 1,84^{\mathrm{b}}$ & 2,73 \\
$a^{*}$ & $0,38 \pm 0,48^{\mathrm{b}}$ & $7,93 \pm 1,13^{\mathrm{a}}$ & $8,28 \pm 1,28^{\mathrm{a}}$ & 18,48 \\
$b^{*}$ & $4,26 \pm 1,25^{\mathrm{b}}$ & $7,35 \pm 1,78^{\mathrm{a}}$ & $7,17 \pm 1,76^{\mathrm{a}}$ & 25,87 \\
$\mathrm{CRA}(\%)$ & $54,51 \pm 0,89^{\mathrm{b}}$ & $57,57 \pm 2,47^{\mathrm{a}}$ & $56,73 \pm 1,62^{\mathrm{a}}$ & 3,17 \\
$\mathrm{FC}\left(\mathrm{kgf.cm}^{-2}\right)$ & $1,92 \pm 0,17$ & $1,95 \pm 0,31$ & $1,92 \pm 0,38$ & 15,92 \\
$\mathrm{PPC}(\%)$ & $32,62 \pm 1,83$ & $31,39 \pm 2,33$ & $34,62 \pm 1,96$ & 6,72 \\
\hline
\end{tabular}

Médias seguidas de letras diferente nas linhas se diferem pelo teste de Tukey $(\mathrm{P}<0,05)$.

$L^{*}=$ Luminosidade; $a^{*}=$ teor de vermelho; $b^{*}=$ teor de amarelo; CRA = capacidade de retenção de água; $\mathrm{FC}=$ força de cisalhamento; $\mathrm{PPC}=$ perda de peso por cocção. 
Rev. Bras. Saúde Prod. Anim., Salvador, v.16, n.3, p.667-677 jul./set.., $2015 \quad \underline{\text { http://www.rbspa.ufba.br }}$ ISSN 15199940

De acordo com Faria et al. (2009) e Warris (2010) há variação no $\mathrm{pH}$ da carne proveniente de músculos de diferentes localizações anatômicas. Os músculos envolvidos com atividade locomotora, como a coxa e a sobrecoxa, por suas atividades biológicas normais (movimentação), geralmente, apresentam menor reserva de glicogênio no miócito para a glicólise post mortem. Desta forma, é comum haver, nestes músculos, acidificação menos extensa e maiores valores de $\mathrm{pH}_{24 \mathrm{~h}}$.

A diferença no $\mathrm{pH}_{24 \mathrm{~h}}$ dos cortes, provavelmente, é a justificativa para as variações na cor, principalmente na luminosidade $\left(L^{*}\right)$ e na capacidade de retenção de água. Na carne do peito, de menor $\mathrm{pH}_{24 h}$ que os demais cortes, há menor hidrofilia das proteínas miofibrilares, a qual ocasionada pela mais intensa neutralização de cargas elétricas superficiais negativas, típicas destas proteínas, pelos prótons hidrogênio $\left(\mathrm{H}^{+}\right)$, naturalmente liberados durante a acidificação post mortem (WARRIS, 2010). Os polos neutralizados perdem a sua capacidade de interagir com a água presente no sarcoplasma, o que ocasiona menor capacidade de retenção de água. Este evento foi observado na carne do peito, a qual apresentou menor $\mathrm{pH}_{24 \mathrm{~h}}$ que as demais e menor capacidade de reter água. A água que se desliga das proteínas miofibrilares pode ser retida no sarcoplasma, caso haja soluto em quantidade suficiente para isto. Caso não haja, a água se descola do meio intracelular para o meio extracelular. Durante esta migração, há aumento da umidade na superfície do corte, o que ocasiona maior luminosidade $\left(L^{*}\right)$ da carne devido à maior reflexão da luz incidente. $\mathrm{Na}$ carne do peito, de menor $\mathrm{pH}_{24 \mathrm{~h}}$, foi observada maior luminosidade que a carne dos demais cortes. Neste contexto, Sanfelice et al.
(2010) verificaram que a diminuição da capacidade da retenção de água ocasionou aumento da luminosidade da carne $\left(L^{*}\right)$, como também observado neste tudo.

Conforme Sterten et al. (2009) e Warris (2010), variações no $\mathrm{pH}$ da carne também podem influenciar a força de cisalhamento e a perda de peso por cozimento, os quais são importantes para a aceitação da carne pelo consumidor e para a sua satisfação no preparo e no consumo. No entanto, embora tenha sido observado menor $\mathrm{pH}_{24 \mathrm{~h}}$ na carne do peito e maior $\mathrm{pH}_{24 \mathrm{~h}}$ na carne da coxa e da sobrecoxa, esta variação não foi suficiente para ocasionar diferença significativa na força de cisalhamento e na perda de peso por cozimento destes cortes.

Observou-se que a carne da coxa e da sobrecoxa apresentaram $(\mathrm{P}<0,05)$ maior teor de vermelho $\left(a^{*}\right)$ e o maior teor de amarelo $\left(b^{*}\right)$ que a carne do peito. Estas diferenças podem ser ocasionadas pela menor luminosidade $\left(L^{*}\right)$ da coxa e da sobrecoxa. Em se havendo menor reflexão da luz incidente, há maior oportunidade para que a luz interaja com os pigmentos cromogênicos presentes na superfície do corte, como a mioglobina e os carotenoides acumulados no tecido adiposo. Além disto, em músculos de regiões associadas à locomoção, como coxa e sobrecoxa, há maior intensidade metabólica e maior necessidade de mioglobina, pigmento este, associado ao transporte intracelular de oxigênio (WARRIS, 2010). Ressalta-se que a mioglobina é um dos principais pigmentos cromogênicos das carnes.

Foi constatada diferença significativa $(\mathrm{P}<0,05)$ para capacidade de retenção de água da coxa e sobrecoxa, no entanto, os valores deste parâmetro para o corte do peito não diferem dos demais. Este parâmetro apresenta relevância para 
Rev. Bras. Saúde Prod. Anim., Salvador, v.16, n.3, p.667-677 jul./set.., $2015 \quad \underline{\text { http://www.rbspa.ufba.br }}$ ISSN 15199940

qualidade da carne, seja ela para o consumo direto ou para industrialização, pois quanto maior a capacidade de retenção de água, menor será a perda durante o armazenamento, a comercialização e o processamento, (GOMIDE et al., 2013).

$\mathrm{O} \mathrm{pH}_{24 \mathrm{~h}}$, a cor $\left(L^{*} a^{*} b^{*}\right)$, a capacidade de retenção de água, a força de cisalhamento e a perda de peso por cocção da carne de frangos da linhagem "pesadão vermelho" não foram influenciados pelos diferentes períodos de jejum alimentar pesquisados. Recomendam-se os tempos de jejum de nove ou 12 horas, nos quais há menor peso do trato gastrointestinal e maior rendimento de carcaça fria. Há diferença nas características de qualidade da carne proveniente do peito, coxa e sobrecoxa de frangos de corte "pesadão vermelho", as quais, decorrentes de especificidades anatômicas, metabólicas e estruturais dos grupamentos musculares. Há, portanto, necessidade de se pesquisar não apenas as características de qualidade da carne do peito, mas também da coxa e da sobrecoxa.

\section{AGRADECIMENTOS}

Os autores agradecem à Fundação de Amparo à Pesquisa do estado de Minas Gerais (FAPEMIG), Coordenação de Aperfeiçoamento de Pessoal de Nível Superior (CAPES), Conselho Nacional de Desenvolvimento Científico e Tecnológico (CNPq) e Financiadora de Estudos e Projetos (FINEP) pelo apoio financeiro.

\section{REFERÊNCIAS}

\author{
AMERICAN MEAT SCIENCE \\ ASSOCIATION - AMSA. Research \\ guidelines for cookery sensory and \\ instrumental tenderness measurement \\ of fresh meat. Chicago, 1995.
}

ASSAYAG JUNIOR, M.S.; PEDROSO, A.C.; FRANCO, S.G.; BODZIAK, S.; SILVA, J.C. Efeito da duração do jejum pré-abate sobre peso corporal de frangos de corte aos 45 dias de idade. Brazilian Journal Veterinary Research and Animal Science, v.42, n.3, p.188-192, 2005.

BARACHO, M.S.;CAMARGO, G.A.; LIMA, A.M.C.; MENTEM, J.F.; MOURA, D.J.; MOREIRA, J.; NÄÄS, I.A. Variables Impacting Poultry Meat Quality from Production to Pre-Slaughter: A Review. Brazilian Journal of Poultry Science, v.8, n.4, p.201-212, 2012.

BARGE, M.T.; DESTEFANIS, G.; TOSCANO, G.P.; BRUGIAPAGLIA, A. Two Reading techniques of the filter paper press method for measuring meat water-holding capacity. Meat Science, v.29, n.2, p,183-189, 1991.

CASTRO, J.B.J.; CASTILLO, C.J.C.; ORTEGA, E.M.M.; PEDREIRA, M.S. Jejum alimentar na qualidade da carne de frangos de corte criados em sistema convencional. Ciência Rural, v.38, n.2, p,470-476, 2008.

DENADAI, J.C.; MENDES, A.A; GARCIA; R.G.; ALMEIDA, I.C.L.; MOREIRA, J.; TAKITA, T.S.; PAVAN, A.C. GARCIA, E.A. Efeito da duração do período de jejum pré-abate sobre rendimento de carcaça e a qualidade da carne do peito de frangos de corte.

Revista Brasileira de Ciência Avícola, v.4, n.2, p.101-109, 2002. 
Rev. Bras. Saúde Prod. Anim., Salvador, v.16, n.3, p.667-677 jul./set.., $2015 \quad \underline{\text { http://www.rbspa.ufba.br }}$ ISSN 15199940

FARIA, P.B.; BRESSAN, M.C.;

SOUZA, X.R.; RODRIGUES, E.C.;

CARDOSO, G.P.; GAMA, L.T.

Composição proximal e qualidade da carne de frangos das linhagens paraíso pedrês e pescoço pelado. Revista Brasileira de Zootecnia, v.38, n.12, p.2455-2464, 2009.

GARCIA, R.G.; CALDARA, F.R.; VARGAS JUNIOR, F.M.; GRACIANO, J.D.; FREITAS, L.W.; SCHWINGEL, A.W.; MARIN, D.; AMADORI, A.H. Jejum alimentar préabate no rendimento e qualidade de carcaça de frangos de corte tipo griller. Agrarian, v.1, n.2, p.113-121, 2008.

GOMIDE, L. A. M.; RAMOS, E. M.; FONTES, P. R. A carne com alimento; Propriedades da carne fresca. In:

Ciência e qualidade da carne: fundamentos. Viçosa, Universidade Federal de Viçosa, 2013. p. 155.

KOMIYAMA, C.M.;MENDES, A.A.; TAKAHASHI, S.E.; MOREIRA, J.; GARCIA, R.G.; SANFELICE, C.; BORBA, H.S.; LEONEL, F.R.; ALMEIDA PAZ, I.C.L; BALOG, A. Chicken Meat Quality as a Function of Fasting Period and Water Spray.

Brazilian Journal of Poultry Science, v.10, n.3, p.179-183, 2008.

MENDES, A.A. Jejum pré-abate em frangos de corte. Revista Brasileira de Ciência Avícola, v.3, n.3, p.199-209, 2001.

PEREIRA, R.E.P.;MARTINS, M.R.F.B.;MENDES, A.A.; ALMEIDA, P.A.Z I.C.L.; KOMIYAMA, C.M.; MILBRADT, E.L.; FERNANDES, B.C. DA S. Effects of pre-slaughter fasting on broiler welfare, meat quality, and intestinal integrity. Brazilian Journal of Poultry Science, v.15, n.2, p.119122, 2013.
RAMÃO, I.B.; NUNES, R.V.; BRUNO, L.D.G.; TSUTSUMI, C.Y.; SILVA, W.T.M.; POZZA, M.S.S. Evaluation of Different Pre-Slaughter Light Intensities and Fasting Duration in Broilers. Brazilian Journal of Poultry Science, v.13, n.4, p.235-240, 2011.

ROSTAGNO, H.S.; ALBINO, L.F.T.; DONZELE, J.L.; GOMES, P.C.; OLIVEIRA, R.F. Tabelas Brasileiras para Aves e Suínos: composição de alimentos e exigências nutricionais. Viçosa, MG: Universidade Federal de Viçosa, 2005.

SANFELICE, C.; MENDES, A.A; KOMIYAMA, C.M.; CAÑIZARES,M.C.; RODRIGUES, L.; CAÑIZARES, G.I.L. Avaliação do efeito do tempo de desossa sobre a qualidade da carne de peito de matrizes pesadas de descarte. Acta Scientiarum Animal Sciences, v.32, n.1, p.85-92, 2010 .

STATISTICAL ANALYSES SYSTEM - SAS. User's guide. Cary: SAS Institue INC., 1996.

SCHETTINO, D.N.; CANÇADO, S.V.; BAIÃO, N.C.; LARA, L.J.C.; FIGUEIREDO, T.C.; SANTOS, W.L.M.Efeito do período de jejum préabate sobre o rendimento de carcaça de frango de corte. Arquivo Brasileiro de Medicina Veterinária e Zootecnia, v.58, n.5, p.918-924, 2006.

STERTEN, H.; FRØYSTEIN, T.; OKSBJERG, N.; REHNBERG, A.C.; EKKER, A.S.; KJOS, N.P. Effects of fasting prior to slaughter on technological and sensory properties of the loin muscle (M. longissimus dorsi) of pigs. Meat Science, v.83, p.351-357, 2009. 
Rev. Bras. Saúde Prod. Anim., Salvador, v.16, n.3, p.667-677 jul./set.., $2015 \quad \underline{\text { http://www.rbspa.ufba.br }}$ ISSN 15199940

VAN LAACK, R.L.J.M.; LIU, C.H.;

SMITH, M.O.; LOVEDAY, H.D.

Characteristics of pale, soft, exudative broiler breast meat. Poultry Science, v.79, n.7, p.1057-1061, 2000.

WARRIS, P.D. Meat science: an introductory text. 2ed. New York: CABI Pub. Inc., 2010.

YANCE, J.W.S.; WHARTON, M.D.; APPLE, J.K. Cookery method and endpoint temperature can affect the Warner-Bratzler shear force, cooking loss, and internal cooked color of beef longissimus steaks. Meat Science, v.88, n.1, p.1-7, 2011.

Data de recebimento: 19/05/20104

Data de aprovação: 20/07/2015 